\title{
Switchable multi-wavelength erbium-doped fiber laser for remote sensing
}

\author{
R. A. Perez-Herrera ${ }^{a}$, S. Diaz ${ }^{a}$, M. Fernandez-Vallejo ${ }^{a}$, M. Lopez-Amo ${ }^{a}$, \\ M. A. Quintela ${ }^{\mathrm{b}}$, J. M. Lopez-Higuera ${ }^{\mathrm{b}}$ \\ ${ }^{a}$ Dept. of Electric and Electronic Engineering, Universidad Publica de Navarra, \\ Campus Arrosadía s/n E-31006 Pamplona, Spain. \\ ${ }^{\mathrm{b}}$ Photonics Engineering Group, University of Cantabria, Cantabria, Spain
}

\begin{abstract}
In this work, we present and experimentally demonstrate a switchable Erbium-doped fiber laser for remote sensing applications. The laser uses four Fiber Bragg Gratings (FBGs) for wavelength selection and for temperature sensing and a $2 \times 4$ optical switch. By adjusting the switch combinations, the laser can be switched among the four different wavelength lasing configurations. Stable one- and two- wavelength oscillations were achieved based on the use of this device. An output power instability analysis with the temperature for two different multi-wavelength configurations, one of them using the $2 \times 4$ optical switch and the other using a $1 \times 4$ coupler, was performed, for a cavity length of $50 \mathrm{~km}$. A comparison between the performances of these topologies is carried out, and their temperature sensitivity and stabilization time are shown.
\end{abstract}

Keywords: switchable multi-wavelength operation, tunable multi-wavelength erbium-doped fiber laser, optical fibre ring laser, fibre Bragg grating (FBG), optical fibre amplifier

\section{INTRODUCTION}

Multiwavelength erbium doped fiber lasers (MEDFL) have attracted attention due to their potential applications in both sensing and telecommunication fields (wavelength-division multiplexing, fiber-optic sensors, optical spectroscopy, etc) [1]. Among the different types of EDFL, the ring laser is a very competitive structure to achieve simultaneously the requirements of many of these applications: narrow linewidth and high output optical power. Nevertheless, the ring fiber lasers are also known to be susceptible to output power instabilities and mode competition [2]. The design parameters optimization of these lasers, such as erbium doped fiber (EDF) length and the coupling ratio can reduce these undesirable effects. Moreover, an appropriate election of a ring laser configuration can improve considerably the characteristics of these lasers [3-4]. In contrast with the FBG sensor system based on a broad-band light source, the advantages of a FBG laser sensor includes its high resolution for wavelength shift and high optical signal-to-noise ratio (SNR) against the noisy environments in practical applications, as remote sensing [5,6]. In this work, the experimental results of the output power stability of a four wavelengths switchable laser for remote sensing are presented.

\section{EXPERIMENTAL SETUP}

In this work, two different parallel EDFRLs configurations are experimentally studied and compared. The experimental setups of proposed EDFRLs are shown in Fig. 1. The first set up comprises a C-band EDFA module, a 2x4 optical switch and a 1x4 optical coupler. We used a compact lightwave electromechanical switch model 86060C from Agilent Technologies and we only utilize one of its inputs. By appropriately adjusting the switch combinations, the ring cavity laser can be made to operate in the single- and dual-wavelength states. The output $2 \times 4$ coupler is used for collecting the four arms signals and for extracting part of the optical output laser from the ring to the output port. Each one of these braches incorporates a FBG for selection of the lasing wavelengths and simultaneously, to simulate a sensor located at $25 \mathrm{~km}$ of distance by means of a $50 \mathrm{~km}$ SMF fiber roll. An optical circulator is used in each branch to insert the FBGs reflected signals into the ring. In this configuration four circulators were used to direct the signal inside the ring, ensuring unidirectional operation and therefore avoiding the spatial hole-burning effect. Because of this, inserting isolators was not necessary. The operation wavelengths of the FBGs have been located in the flat region of the erbium 
gain profile in order to achieve good equalization between channels. In this test, gratings of 1531.12, 1535.77, 1539.62, and $1545.23 \mathrm{~nm}$ have been used, each one showing about $0.3 \mathrm{~nm}$ at $-3 \mathrm{~dB}$ bandwidth and about $97 \%$ of reflectivity. All the free terminations of the system have been immersed in refractive-index-matching gel to avoid undesired reflections.

By using this structure we can select the emission wavelength of the laser, obtaining better output power levels, OSNRs and stability in comparison with a non-switchable multiwavelength laser. We also avoid the utilization of variable optical attenuators (VOA) that are usually necessary in EDFRLs which emit simultaneously more than two wavelengths. Indeed, with this switch we can also allow a dual wavelength emission without VOAs. The price to pay is a reduction of the output power and stability of the selected wavelengths. Fig 2 (b) and (c) represent some typical examples of the lasing output spectra of this configuration.

In order to check the improvement of the OSNR and the output power level of this structure, the results for using a $1 \times 4$ optical coupler instead an optical switch, which allows the simultaneous lasing of the four wavelengths selected by the FBGs, were also studied and analyzed. The experimental setup for this second EDFRL is shown in Fig. 1(b) and the emission spectrum of the laser is shown in Fig 2(a). One of the major problems in multiwavelength ring lasers is correctly adjusting the cavity losses on each wavelength in order to achieve oscillation of the system in all the desired channels. The oscillation threshold power for each wavelength is different due to the nonflat shape of the erbium fiber gain profile; as a consequence, individual loss control for each wavelength is required. In order to resolve this problem, a variable optical attenuator has been connected to each FBG in this second configuration.

An output power instability study at room temperature and an optical signal-to-noise (OSNR) comparison of these configurations (single-, dual or four-wavelength lasing operation) was carried out. The spectral characteristics of the lasers are measured using an optical spectrum analyzer (OSA) at the output port with $0.1 \mathrm{~nm}$ resolution.

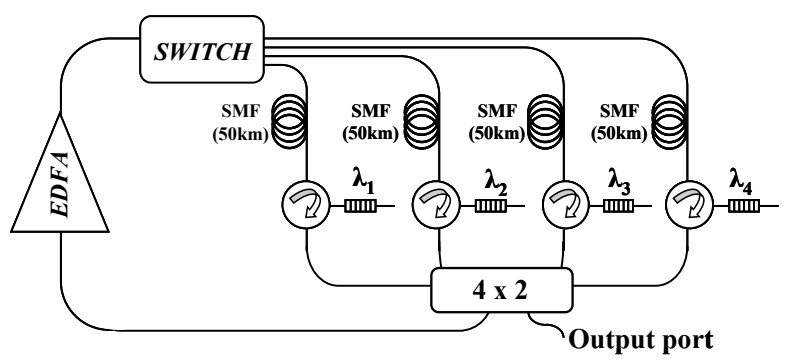

(a)

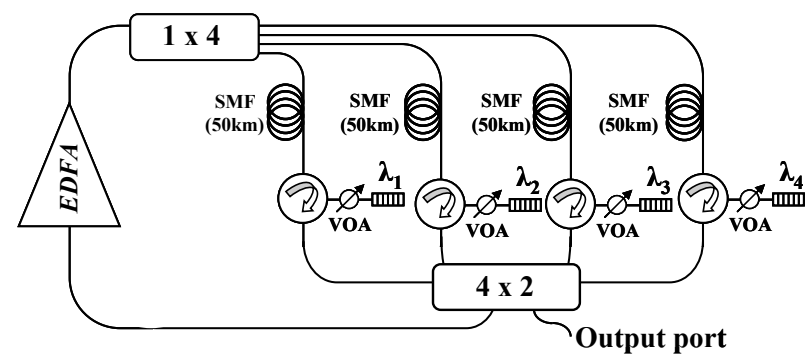

(b)

Fig.1. Experimental set-ups of the multiwavelength EDF ring lasers by using a $2 \times 4$ optical switch (a) or by using a $1 \times 4$ coupler (b)

\section{EXPERIMENTAL RESULTS AND DISCUSSIONS}

By using the set up shown in Fig 1 (b), four lasing wavelengths are obtained simultaneously. The output spectrum of the EDFRL for a $90 \mathrm{~mW}$ pump power is shown in Fig. 2(a). The power of each of the four output channels is around -50 $\mathrm{dBm}$, and the power difference between the four channels (obtained using VOAs) is around $0.9 \mathrm{~dB}$. The four independent lasing wavelengths show an OSNRs (measurement of the ratio of signal power to noise power in an optical channel) of around $32 \mathrm{~dB}$. However, both the output power and OSNR improve when the optical coupler is replaced by an optical switch (see figures 2 (b) and (c)). In this case only by adjusting the switch combinations, the laser can be switched among the four different wavelength lasing one by one or two by two (one or two at a time). Furthermore, variable optical attenuators (VOA) were not needed because of the switch operation. By selecting just one wavelength we also increase the output power and stability, because we eliminate the gain competition among the different wavelengths.

As can be seen in Fig 2 (b), a single-wavelength laser with an output power of around $-10 \mathrm{~dB}$ and OSNRs of more than $62 \mathrm{~dB}$ can be obtained. This figure shows only one of the four possible lasing wavelengths that can be generated by doing that, all of them showing the same characteristics. Finally, Fig. 2(c) corresponds to a dual-wavelength switch operation, showing one of the four possible lasing wavelengths combinations. The power obtained from each of the output channels is around $-17 \mathrm{dBm}$ and the two lasing wavelengths show OSNRs around $58 \mathrm{~dB}$. 
In order to investigate the applicability of the proposed remote sensing system, the output power stability of these structures was also analyzed. Thus, one of the FBGs was placed inside a climatic chamber and temperature cycles from $30^{\circ} \mathrm{C}$ to $100^{\circ} \mathrm{C}$ were carried out. The instability was defined as the output power variations (measured in $\mathrm{dB}$ ) for a given interval of temperature and a specific confidence level (CL), that is the probability value associated with a confidence interval, given as a percentage [7]. In these experiments a CL of $99 \%$ was used.

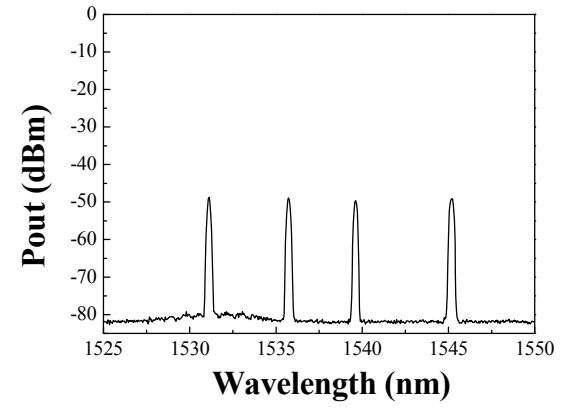

Fig. 2. (a) Output spectrum for the fourwavelength lasing configuration

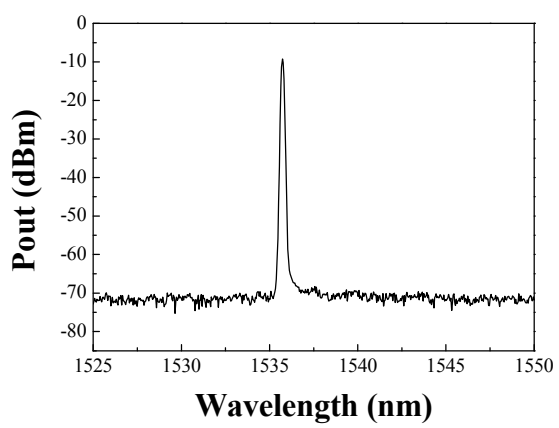

Fig. 2. (b) Output spectrum for the singlewavelength lasing configuration

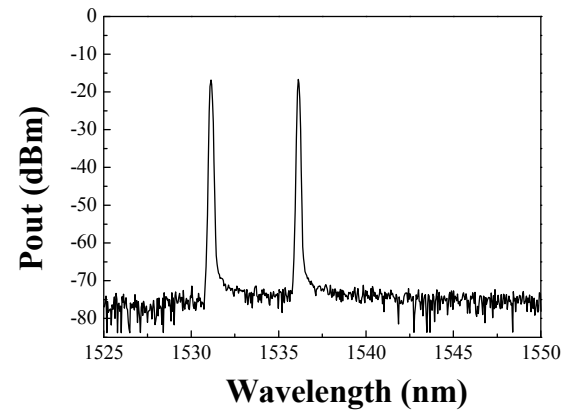

Fig. 2. (c) Output spectrum for the dualwavelength lasing configuration

Fig. 3 (a) and (b) show the central wavelength shift of each FBG of the four-wavelength lasers simultaneously and the four single wavelength lasers, one by one, when temperature increases. Inset the figure, the equations that relate each lasing wavelength with the temperature, the mean square error and the output power fluctuations are presented.

For the first configuration, as can be seen in Fig 3 (a), the center wavelength shift for the four-wavelength lasers presents a clear linear behavior, and the temperature sensitivity ranges from $10 \mathrm{pm} /{ }^{\circ} \mathrm{C}$ for the worst case to $7 \mathrm{pm} /{ }^{\circ} \mathrm{C}$ for the best one. The measured output power instabilities were among $7.36 \mathrm{~dB}$ to $0.6 \mathrm{~dB}$, for the worst case and for the best one respectively. A similar experimental study for the single-wavelength laser was carried out. Its temperature sensitivity has an analogous behavior ranging from $11 \mathrm{pm} /{ }^{\circ} \mathrm{C}$ to $7 \mathrm{pm} /{ }^{\circ} \mathrm{C}$. However the output power instabilities were between 0.66 $\mathrm{dB}$ to $0.47 \mathrm{~dB}$, for the worst and the best case respectively, showing a great improvement in these obtained results. Finally, this output power study was also carried out for the dual-wavelength laser, showing output power fluctuations from $0.66 \mathrm{~dB}$ to $3.91 \mathrm{~dB}$.

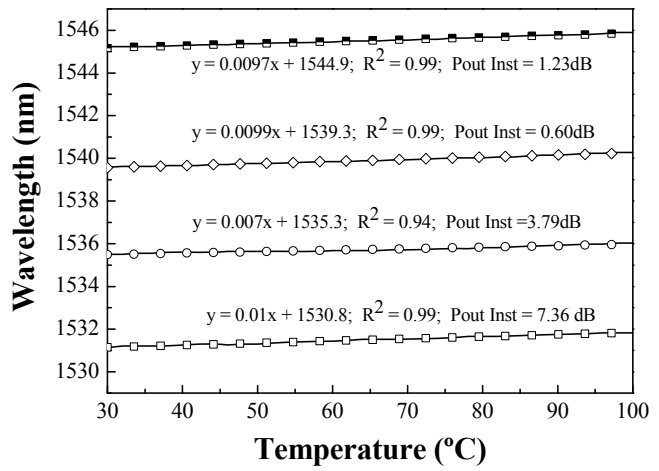

Fig. 3. (a) Wavelength shift of each FBG against temperature for the fourwavelength lasing operation

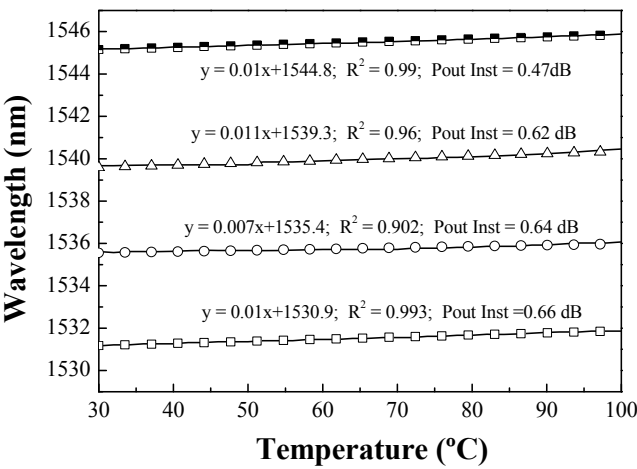

Fig. 3. (b) Wavelength shift of each FBG against temperature for the single-wavelength lasing operation

If a comparison between these results is carried out, we can conclude that the structures which utilize an optical switch are better in terms of output power stability than the first one but, just as it was expected, when the number of laser wavelengths increases, the output power instability increases as well.

We have also measured the switching and stabilization time both for single and dual wavelength operation. Fig 4 (a) and (b) show both the switching and the stabilization time for these two options. When only one output lasing wavelength is generated, a switching and stabilization time of about $130 \mathrm{~ms}$ and $12.5 \mathrm{~ms}$ were measured. These values 
are shown in Fig 4(a). However, when a dual-wavelength lasing emission is generated, these values increase to $135 \mathrm{~ms}$ and $14.5 \mathrm{~ms}$ respectively, as can be observed in Fig 4(b).

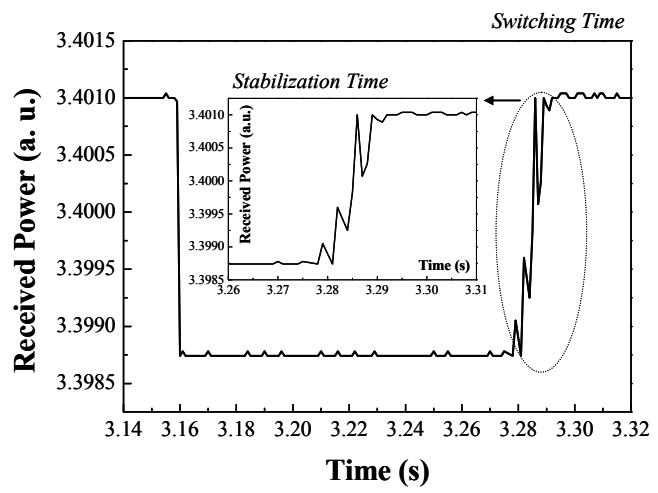

Fig. 4. (a) Switching and stabilization time for the single-wavelength lasing operation

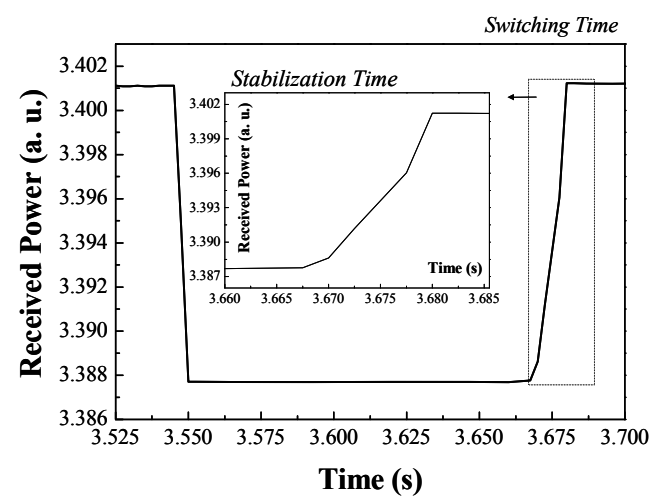

Fig. 4. (b) Switching and stabilization time for the dual-wavelength lasing operation

\section{CONCLUSIONS}

In conclusion, a four-wavelength switchable laser for remote sensing operation has been demonstrated. By appropriately adjusting the possible combinations of a $2 \times 4$ optical switch, the ring cavity laser can be made to operate in the single-, dual- or four-wavelength states. We have characterized its sensitivity, power stability and OSNR for temperature sensing using a $50 \mathrm{~km}$ fiber roll to simulate a remote measurement. We have also compared its behaviour when the switch is replaced by a $1 \times 4$ coupler. We can conclude that the structure with an optical switch is considerably better in terms of output power, stability and OSNR than the non-switchable one.

\section{ACKNOWLEDGEMENTS}

Financial support from the Spanish Comisión Interministerial de Ciencia y Tecnología within project TEC200767987-C02-02, and FEDER funds is acknowledged.

\section{REFERENCES}

[1] L. Talaverano, S. Abad, S. Jarabo, and M. López-Amo, "Multiwavelength fiber laser sources with Bragg-Grating sensor multiplexing capability," Journal of Lightwave Technology, vol. 19, n. 4, 553-558, 2001.

[2] A. Zhang, H. Liu, M. S. Demokan, H. Y. Tam, "Stable and broad bandwidth multiwavelength fiber ring laser incorporating a highly nonlinear photonic crystal fiber," IEEE Photonics Technology Letters, vol. 17, n. 12, 2535-2537, 2005.

[3] R. A. Perez-Herrera, M. A. Quintela, et all, "Stability comparison of two ring resonator structures for multiwavelength fiber lasers using highly doped Er-fibers," accepted for publication in Journal of Lightwave Technology, (2009).

[4] A. Gusarov and F. Liegeois, "Experimental study of a tunable fiber ring laser stability," Optics Communications, vol. 234, n. 1-6, 391-397, 2004.

[5] A. D. Kersey, M. A. Davis, H. J. Partrick, M. Leblance, K. P. Koo, C. G. Askins, M. A. Putnam, and E. J. Friebele, "Fiber grating sensors," Journal of Lightwave Technology, vol. 15, n. 8, 1442-1463, 1997.

[6] P. C. Peng, H. Y. Tseng and S. Chi, "Long-distance FBG sensor system using a linear-cavity fiber Raman laser scheme," IEEE Photonics Technology Letters, vol. 16, n. 2, 575-577, 2004.

[7] http://www.stats.gla.ac.uk/steps/glossary/confidence intervals.html 